\title{
The Methodology and Electro-physiological Classification of Pre-operative Trans-tympanic Electrically-Evoked Auditory Brainstem Response (TT-EABR)
}

\author{
Sunil Narayan Dutt ${ }^{1} \cdot$ Apurva Kumar $^{2}$
}

Published online: 28 January 2019

(C) Association of Otolaryngologists of India 2019

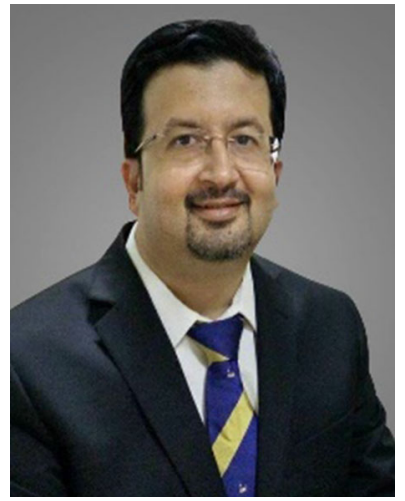

implant surgery than for ears which had abnormal or absent TT-EABR [2].

\section{Pre-operative TT-EABR Methodology}

The authors have used the technique previously described by Gibson and Sanli [2].

\section{Stimulus Side}

Under a short general anesthesia, a posterior segment myringotomy is performed to visualize the round window niche. A subdermal needle electrode is placed under the skin on the ipsilateral temporal region to act as the ground electrode. Biphasic current pulses are delivered through a golf club electrode designed to stimulate the round window membrane at the round window niche (Fig. 1) [2]. Custom Sound EP software (Cochlear Corporation, Australia) with EABR mode is used through the "implant in a box" interfaced via a sound processor. Stimuli at the rate of $11 / \mathrm{s}$, pulse width (PW) of 100-150 $\mu$ s with basic, alternating and reverse polarity are used.

effective clinical procedure which can decrease the likelihood of placing a cochlear implant in a non-stimulable ear, and may provide the clinician with a valuable tool for selection of the most appropriate ear for implantation [1]. It has been shown that the presence of normal TT-EABR may indicate a significantly better outcome after cochlear

Sunil Narayan Dutt

sunildutt@hotmail.com

1 Department of ENT, Apollo Hospitals, Opposite IIM

Bangalore, Bengaluru 560076, India

\section{Recording Side}

A conventional Electrophysiology (EP) GSI-Audera machine with surface electrodes is used for recording the waveforms. The electrode montage used is shown in Fig. 2. Wave $\mathrm{V}$ morphology, latency and repeatability are the parameters recorded.

The whole procedure takes about $20 \mathrm{~min}$ on each side and can be performed as a day care procedure. The myringotomy heals in 2 weeks time in most patients.

2 Ashadeep ENT Centre, Bengaluru, India 


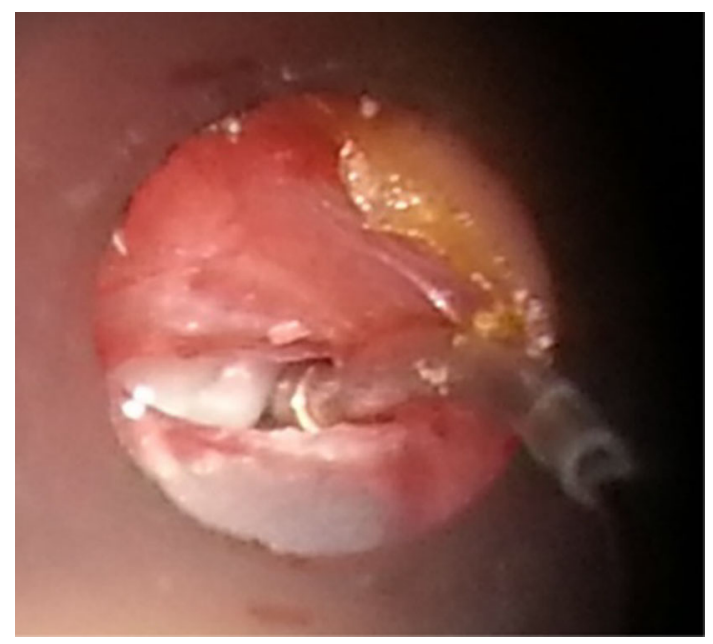

Fig. 1 Golf club electrode placed inside the round window niche (right ear) with the tip directed towards the round window membrane

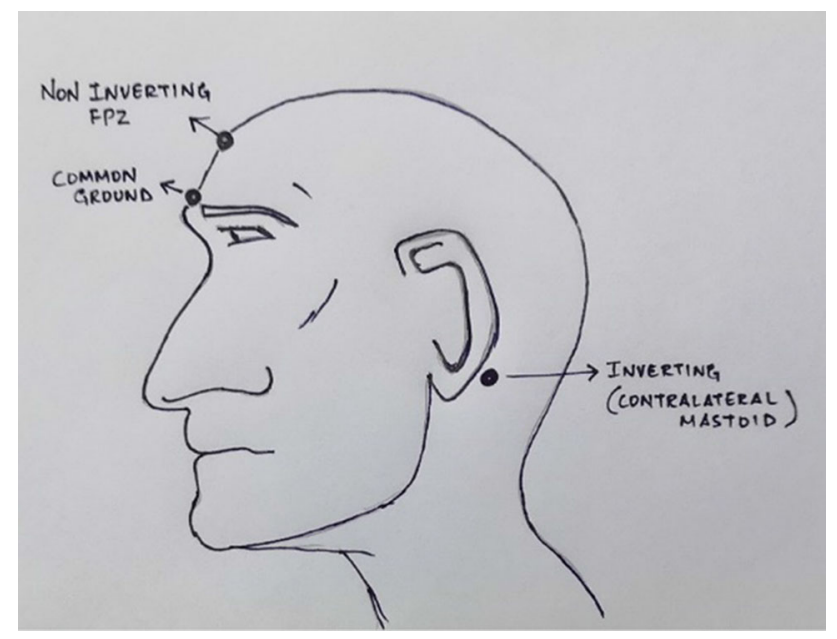

Fig. 2 Montage of the surface electrode placements

\section{Kumar-Dutt Classification of Reporting Responses of TT-EABR}

The authors propose a combined morphological and electrophysiological classification (Kumar-Dutt classification) of reporting responses with TTEABR, thus Type-A, B, C and $\mathrm{D}$ are shown in Fig. 3a-d respectively and described in Table 1.

\section{Discussion}

In today's era of advanced radiology and hence advanced diagnosis of anatomical anomalies of the cochleo-vestibular system, it is important to identify the patients in whom a cochlear implantation would help in improving the hearing abilities. The above proposed classification system provides an objective method for establishing the stimulability of the cochlear nerve in such patients.

Polterauer et al. [3] have described a similar procedure for establishing cochlear implant candidacy in individual where the acoustic auditory brain stem response audiometry did not show reproducible recording even at very high acoustic levels. They compared the Transtympanic EABR with the intra-operative EABR measurements that were stimulated using the implanted CI electrode array and found similar wave forms.

Several researchers including Kileny et al. [4] have described the use of TTEABR in special cases of congenital temporal bone malformation to establish the functional status of the auditory pathway before implantation. However, no system has been described to classify the responses obtained. Kumar-Dutt classification aims to fill this gap in our knowledge of TTEABR and hence improve the reportability of responses obtained. 
Fig. 3 a Type A-Robust Wave V Morphology;

repeatable peak III and $\mathrm{V}$ with peak V latency within $5 \mathrm{~ms}$.

b Type B-Good Wave V

Morphology; repeatable peak V

(and not III), with latency

within 5 ms. c Type C-Fair/

acceptable Wave $\mathrm{V}$

Morphology; peak V present

with delayed latency (beyond

$5 \mathrm{~ms}$ ). d Type D-Poor or

Absent Wave V $\mathbf{a}$

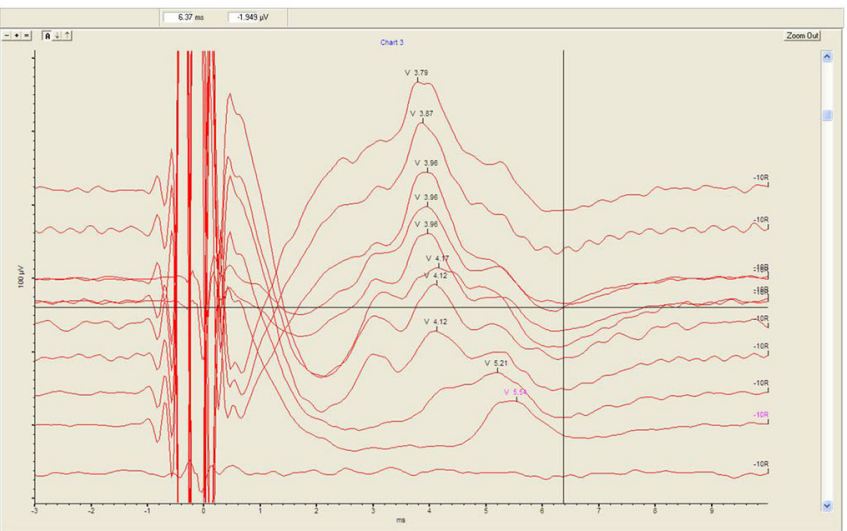

b

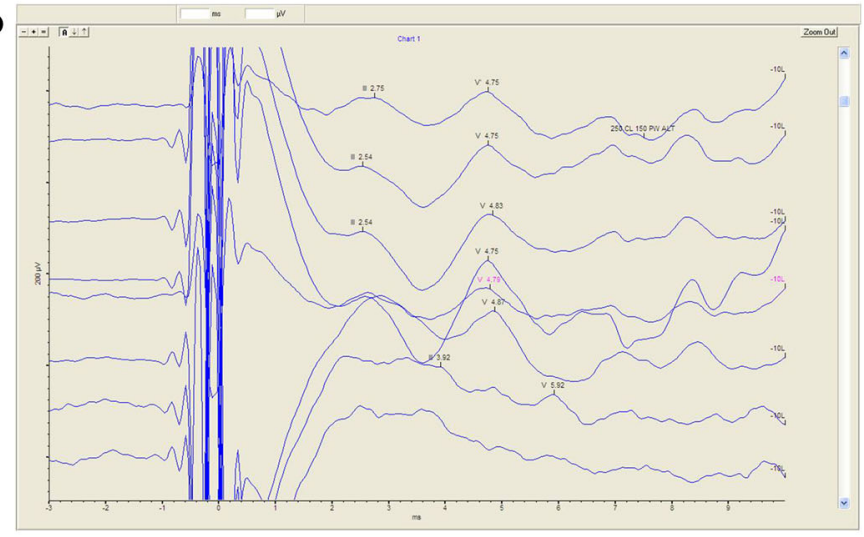

c

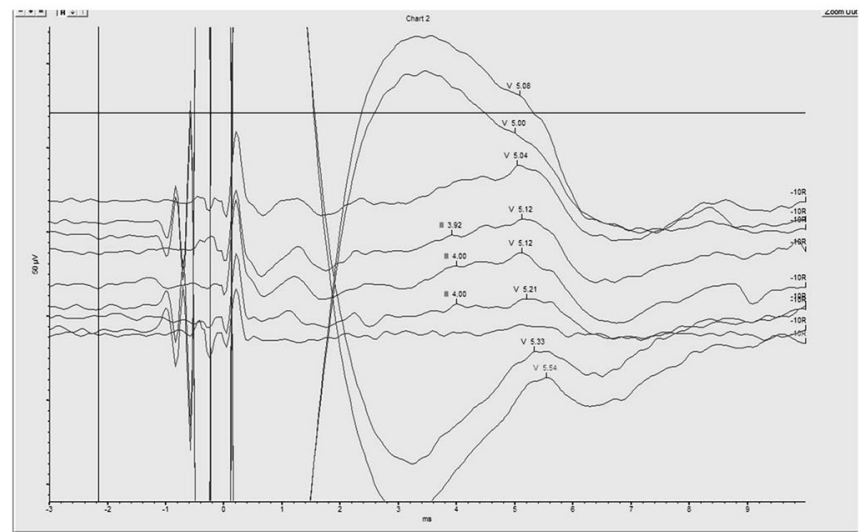

d

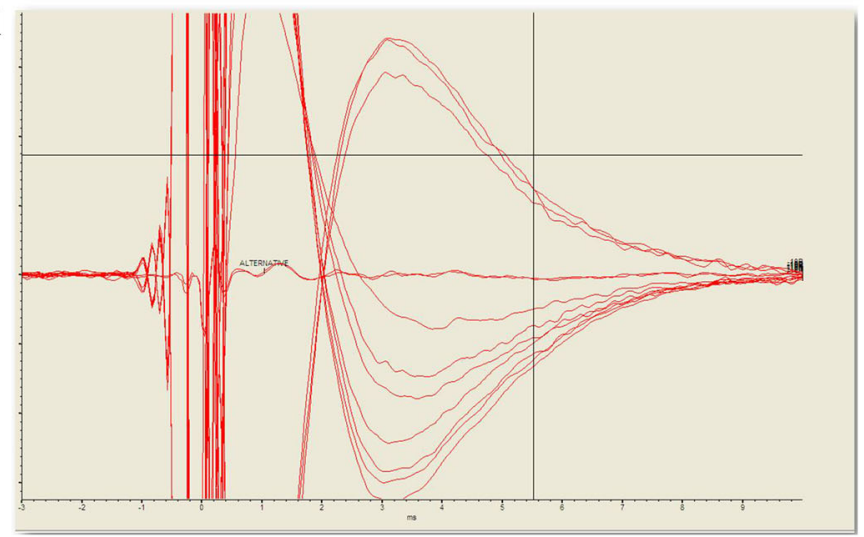


Table 1 Description of the Wave Form Types A, B, C and D according to the Kumar-Dutt electrophysiological classification for TT-EABR

\begin{tabular}{ll}
\hline Type & Description \\
\hline A & Robust Wave V Morphology; repeatable peak III and V with peak V latency within 5 ms \\
B & Good Wave V Morphology; repeatable peak V (and not III), with latency within 5 ms \\
C & Fair/acceptable Wave V Morphology; peak V present with delayed latency (beyond 5 ms) \\
D & Poor or Absent Wave V \\
\hline
\end{tabular}

\section{References}

1. Kileny PR, Zwolan TA (2004) Pre-perioperative, transtympanic electrically evoked auditory brainstem response in children. Int $\mathbf{J}$ Audiol 43(Suppl 1):S16-S21

2. Gibson W, Sanli H (2007) Auditory neuropathy: an update. Ear Hear 28(Supplement):102S-106S. https://doi.org/10.1097/aud. 0b013e3180315392

3. Polterauer D, Neuling M, Müller J, Hempel J, Mandruzzato G, Polak M (2018) PromBERA: a preoperative eABR: an update. Curr Dir Biomed Eng 4(1):563-565. https://doi.org/10.1515/ cdbme-2018-0135
4. Kileny P, Kim A, Wiet R, Telian S, Arts H, El-Kashlan H, Zwolan $\mathrm{T}$ (2010) The predictive value of transtympanic promontory EABR in congenital temporal bone malformations. Cochlear Implants Int 11(sup1):181-186.

https://doi.org/10.1179/146701010x12671177818669

Publisher's Note Springer Nature remains neutral with regard to jurisdictional claims in published maps and institutional affiliations. 